\title{
Numerical analysis of MHD free convection boundary layer flow of heat and mass transfer in case of air and water
}

\author{
M. Ali $* 1$, M. S. Alam ${ }^{1}$, M. A. Alim² \\ ${ }^{1}$ Chittagong University of Engineering and Technology (CUET), Chittagong-4349, Bangladesh \\ ${ }^{2}$ Bangladesh University of Engineering and Technology (BUET), Dhaka-1000, Bangladesh
}

\begin{abstract}
The present work has been studied the effect of various parameters on MHD boundary layer flow of heat and mass transfer in case of air and water. The governing partial differential equations are transformed to higher order ordinary differential equations by using similarity transform. This coupled ordinary differential equations are performed by shooting iteration technique along with Runge-Kutta integration scheme. The effects of various parameters on velocity, temperature and concentration profiles are discussed numerically and shown graphically. Therefore, the results of velocity field decreases for increasing values of magnetic parameter and chemical reaction parameter in case air and salt water but increases for Grashof number and modified Grashof number. The temperature field decreases in the presence of magnetic parameter but significant increasing effect for chemical reaction parameter, Grashof number and modified Grashof number in case of air and salt water. Also, the concentration profile is slightly increased for increasing the values of magnetic parameter and slightly decreased for Grashof number and modified Grashof number but significant decreasing effect are observed for reaction parameter. Finally, the numerical values of the shear stress, rate of temperature and rate of concentration are also shown in a tabular form.
\end{abstract}

Keywords: MHD; Heat absorption; Chemical reaction; Free convection

\section{Introduction}

Heat and mass transfer analysis with magnetic field has a great interest to engineers and scientist because of its universal applications of many branches of science and engineering. In many chemical processes, a chemical reaction occurs between a foreign mass and a fluid in which a plate is moving. These processes has numerous applications such as polymer production, manufacturing of ceramics or glassware and food processing. Besides, the magneto hydrodynamic laminar boundary layer behavior over a stretching surface is a significant type of flow having considerable practical applications in chemical engineering, electrochemistry and polymer processing. This problem has also an important bearing on metallurgy where magneto hydrodynamic (MHD) techniques have recently been used. In this regard, Abel and Mahesh (2008) presented an analytical and numerical solution for heat transfer in a steady laminar flow of an incompressible viscoelastic fluid over a stretching sheet with power-law surface temperature, including the effects of variable thermal conductivity and non-uniform heat source and radiation. The effect of chemical reaction on free-convective flow and mass transfer of a viscous, incompressible and electrically conducting fluid over a stretching sheet was investigated by Afify (2004) in the presence of transverse magnetic field. Alam et al. (2014) discussed the steady MHD boundary layer free convective heat and mass transfer flow over an inclined porous plate with variable suction and soret effect in presence of Hall current, Ali et al. (2010) have analyzed the MHD viscos flow of heat transfer over a permeable shrinking sheet with surface heat flux, Cortell (2005) studied the magneto hydrodynamics flow of a power-law fluid over a stretching sheet, Iskak et al. (2008) discussed the heat transfer analysis for mixed convection flow through a vertical stretching sheet, later Iskak et al.(2006) also analyzed the heat transfer of unsteady mixed convection flow over vertical stretching sheet, Rajesh (2010) have been studied the MHD effect on free convection and mass transform flow through a porous medium with variable temperature, Sing (2012) studied the heat and mass transfer in MHD boundary layer flow past an inclined plate with viscous dissipation in porous medium, Sonth et al.(2002) discussed heat and mass transfer in a visco-elastic fluid flow over an accelerating surface with heat source/sink and viscous dissipation and Tan et

\footnotetext{
*Corresponding author. e-mail: ali.mehidi93@gmail.com
} 
al. (2008) studied heat and mass transfer over an impermeable stretching plate. Since the study of heat and mass transfer is important in many cases, in the present paper we studied the free convection heat and mass transfer flow over a semi-infinite vertical plate with heat absorption, chemical reaction and magnetic field in case of air and water. The boundary layer equations are transformed by a similarity transformation into a system of coupled non-linear ordinary differential equations and which are then solved numerically by shooting iteration technique along with Runge- Kutta sixth order method.

\section{Governing Equations of the Present Problem}

The flow is considered along the axis of $\mathrm{x}$ and the magnetic field is applied to the perpendicular of the direction of fluid flow. Also the flow is electrically conducting and steady laminar two dimensional. The uniform plate temperature $\mathrm{T}_{\mathrm{w}}$ $\left(>T_{o o}\right)$, where $T_{o o}$ is the temperature of the fluid far away from the plate. Let $\mathrm{u}$ and $\mathrm{v}$ be the velocity components along the $\mathrm{x}$ and $\mathrm{y}$ axis respectively in the boundary layer region. Under the above assumptions and usual boundary layer approximation, the dimensional governing partial differential equations of continuity, momentum, energy and concentration under the influence of externally imposed magnetic field are [2]:

\section{Equation of continuity:}

$\frac{\partial x}{\partial x}+\frac{\partial v}{\partial y}=0$

Momentum equation

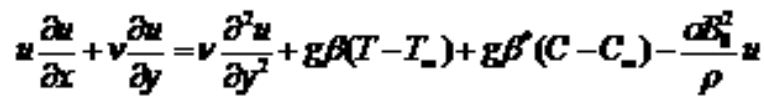

Energy Equation

$v \frac{\partial T}{\partial x}+v \frac{\partial T}{\partial y}=\frac{x}{\rho c_{p}} \frac{\partial^{2} T}{\partial y^{2}}+Q^{*}\left(\mathrm{C}-\mathrm{C}_{-}\right)$

Concentration Equation

$v \frac{\partial C}{\partial x}+v \frac{\partial C}{\partial y}=D_{-} \frac{\partial^{2} C}{\partial y^{2}}-x\left(C-C_{n}\right)$
Boundary conditions are

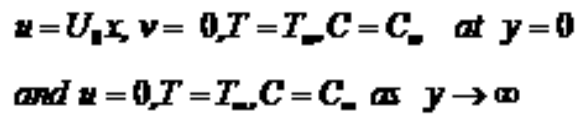

where $\mathrm{u}$ and $\mathrm{v}$ are the velocity components along $\mathrm{x}$ and $\mathrm{y}$ directions, $\mathrm{T}, \mathrm{T}_{\mathrm{w}}$ and $\mathrm{T}_{\mathrm{oo}}$ are the fluid temperature, the plate temperature and the free stream temperature respectively while $\mathrm{C}, \mathrm{C}_{\mathrm{w}}$ and $\mathrm{C}_{\mathrm{oo}}$ are the corresponding concentrations, $\mathrm{a}$ is the constant, $k$ is the thermal conductivity, $\lambda^{*}$ is the reaction rate constant, $Q^{*}$ is the heat absorption rate constant, $\mathrm{q}$ is the heat flux, $C_{p}$ specific heat with constant pressure, $v$ is the kinematic viscosity, $\sigma$ is the electrical conductivity, $\rho$ is the fluid density, $\beta$ is the thermal expansion coefficient, $\beta^{*}$ is the concentration expansion coefficient, $B_{o}$ is the magnetic field intensity, $g$ is the acceleration due to gravity, $D_{m}$ is the coefficient of mass diffusivity respectively. We introduce the steam function $\psi(x, y)$ as defined by

$v=\frac{\partial r}{\partial y} \rightarrow v=-\frac{\partial r}{\partial x}$

To convert the governing equations into a set of coupled ordinary differential equations, we take the following similarity transformation:

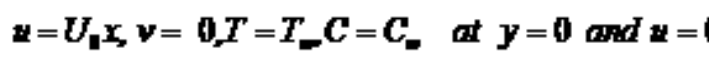

$0, T=T_{-}, C=C_{-} \sigma$

From the above transformations, the non-dimensional, nonlinear and coupled ordinary differential equations are obtained as

$$
\begin{aligned}
& f^{*}+\frac{1}{2} f^{\prime}-M f^{\prime}+G_{r} \theta+G_{m} \phi=0 \\
& \theta^{*}+\frac{1}{2} P\left(\theta^{\prime}-f^{\prime} \theta\right)+\operatorname{PrQ} \varphi=0 \\
& \varphi+\frac{1}{2} \operatorname{ser}\left(\varphi^{\prime}-f \varphi\right)-\sec _{\varphi}=0
\end{aligned}
$$

The transform boundary conditions $z=U_{1}, v_{2}=0, T=T_{-} C=C_{-}$at $y=0$ ard $z=0, T=T_{-} C=$

Where $f, \theta$ and $\varphi$ are the dimensionless velocity, temperature and concentration profiles respectively, $\eta$ is the similarity 
variable, the prime denotes differentiation with respect to $\eta$. Also the non-dimensional parameters are

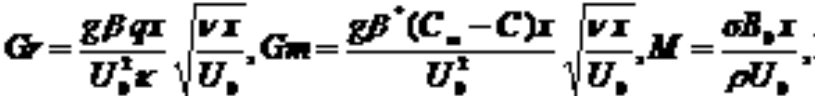

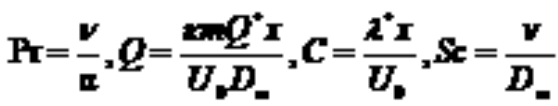

This are denoting the Grashof number, modified Grashof number, magnetic parameter, Prandtl number, heat absorption parameter, chemical reaction parameter and Schmidt number respectively.

\section{Results and discussion}

Numerical calculation for distribution of the velocity, temperature and concentration profiles across the boundary layer are displayed in Fig. 1- Fig.24 for different values of magnetic parameter M, and chemical reaction parameter C, Schmidt number Sc, heat absorption parameter R, Grashof number Gr $\&$ modified Grashof number $\mathrm{Gm}$ in case of air and water. The values of Prandtl number Pr are chosen for 1.00 and
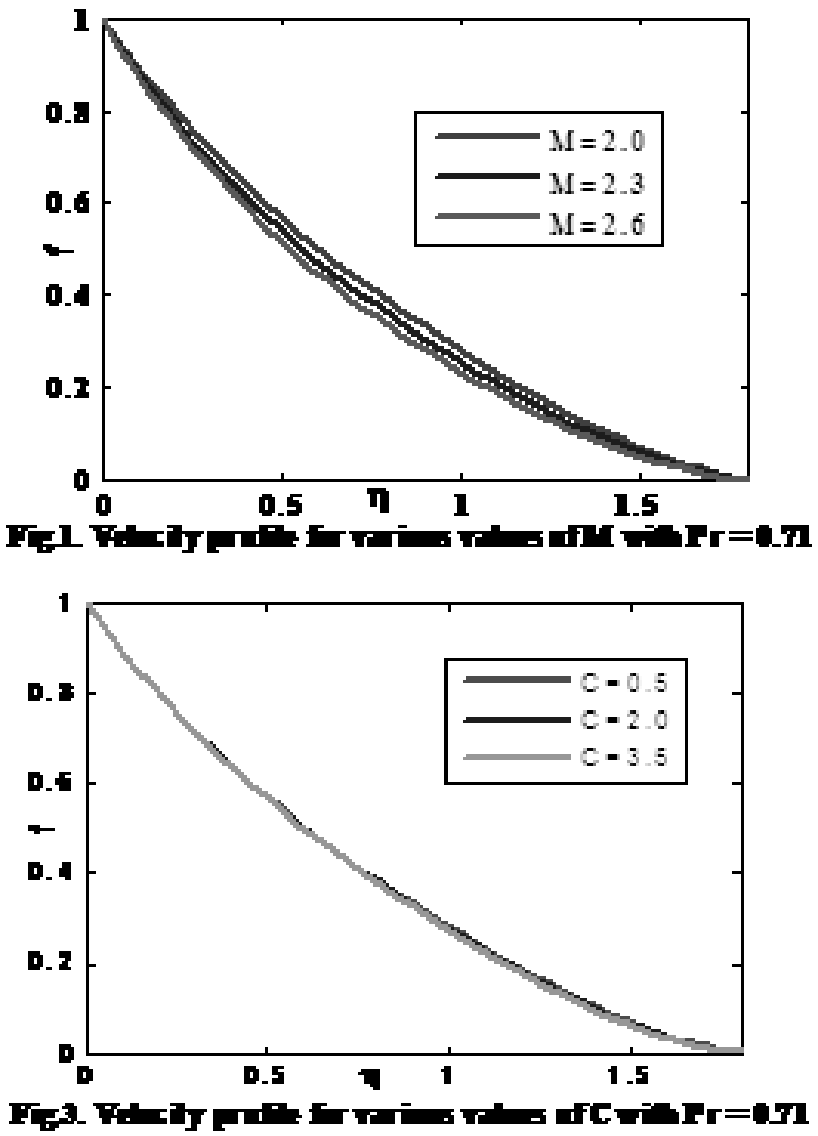

0.71 which correspond to salt water and air and also Schmidt number are taken 0.60 which correspond to water vapor. The effects of various parameters on velocity profile are shown in Fig. 1- Fig. 8. In Fig. 1 it is observed that the velocity decreases with an increase in the M. The magnetic parameter is found to retard the velocity at all points of the flow field. It is because that the application of transverse magnetic field will result in a resistive type force (Lorentz force) similar to drag force which tends to resist the fluid flow and thus reducing its velocity. Similar effect arises for the increasing values of magnetic parameter [Fig. 2] in case of salt water but negligible decreasing effect are found both the cases of air and salt water for increasing values of reaction parameter which are shown in Fig.3 and Fig.4. Again the velocity profile is increased up to certain values of eta and decreased for increasing the values of $\mathrm{Gr}$ in case of air and water which are shown in Fig.5 and Fig.6. Also the profile is increased for $\mathrm{Gm}$ both of air and water are shown in Fig.7 and Fig.8. The effect of various parameters on temperature profile are shown in Fig.9 - Fig.16. From these figures we
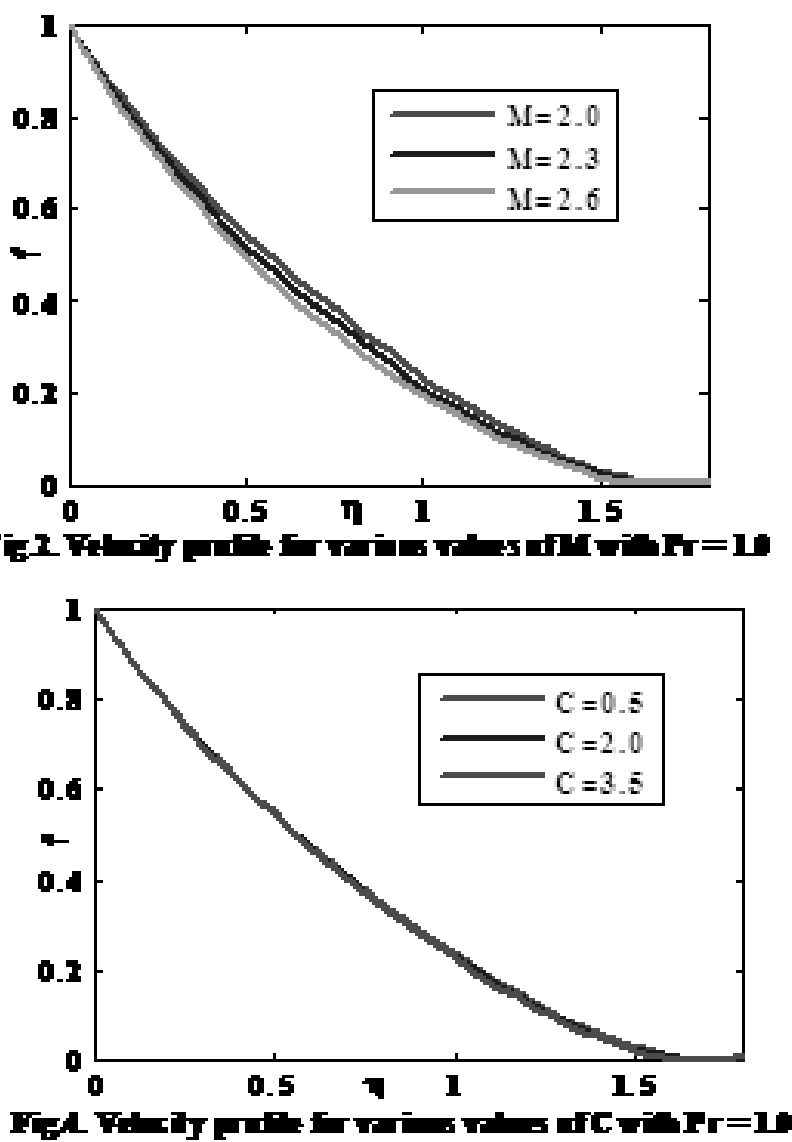
see that, the temperature profile is starting at the initial point of the plate surface and then increasing until it reaches to a maximum point and then decreases far away from the plate satisfying the boundary condition. Fig.9 shows the tempera ture distribution for different values of the magnetic field parameter $\mathrm{M}$ and observed that the thermal boundary layer

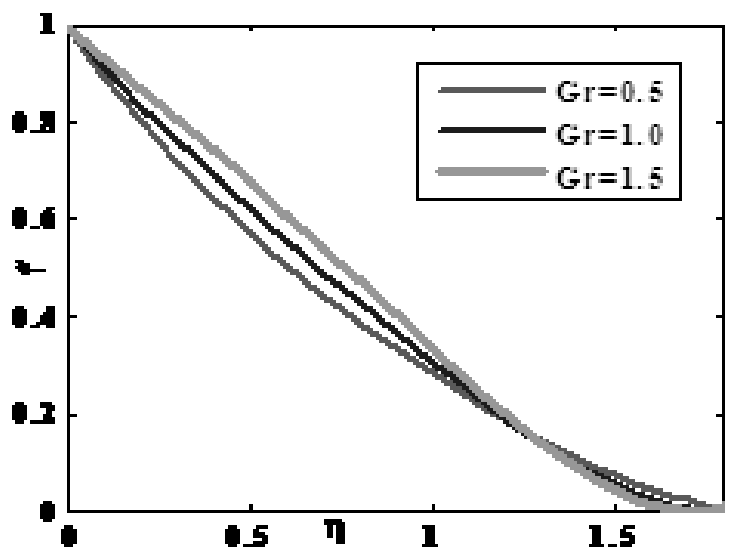

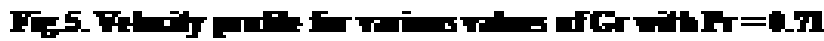
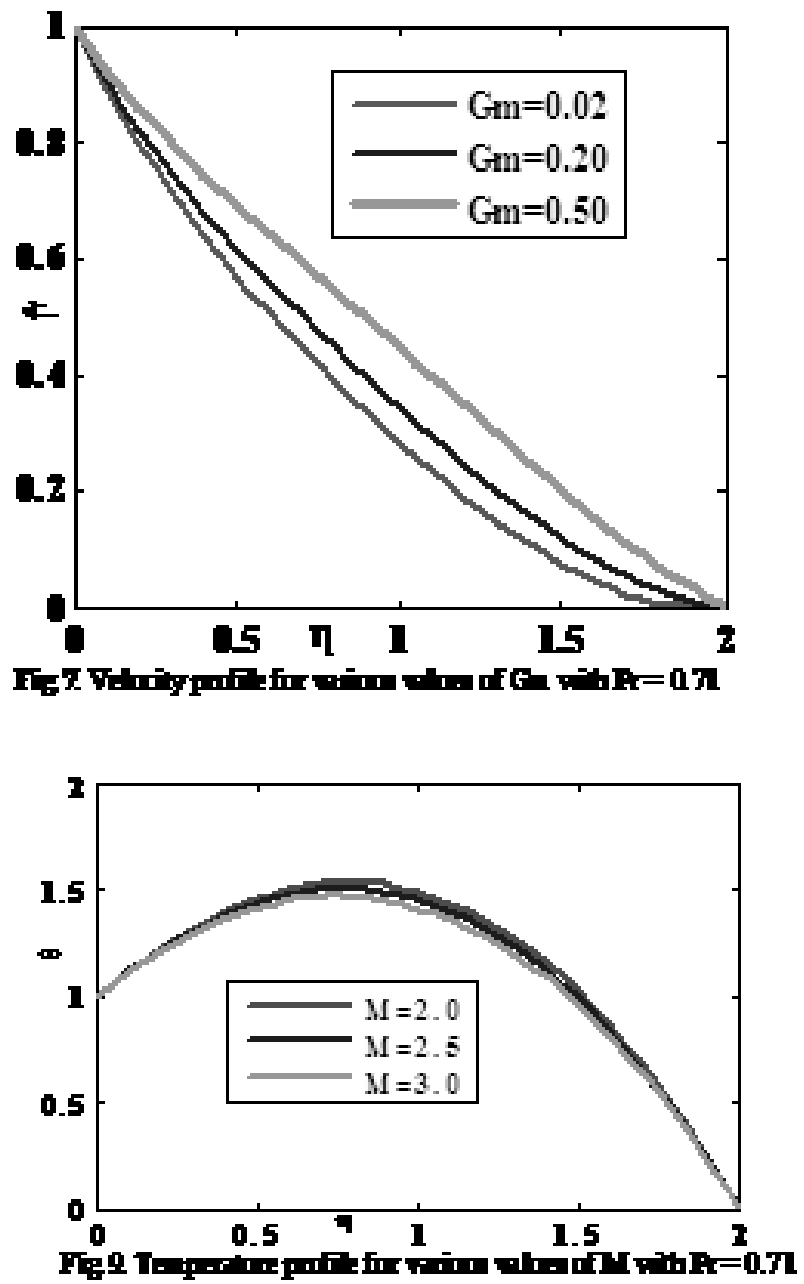

decreases as $\mathrm{M}$ increases adjacent to the surface of the plate and the effect is not significant far away from the plate. Similar result has been found in case of salt water but the rate of heat transfer is greater than in case of air which is shown in Fig. 10 but reverse result arises for the increasing values of chemical reaction, Grashof number and modified

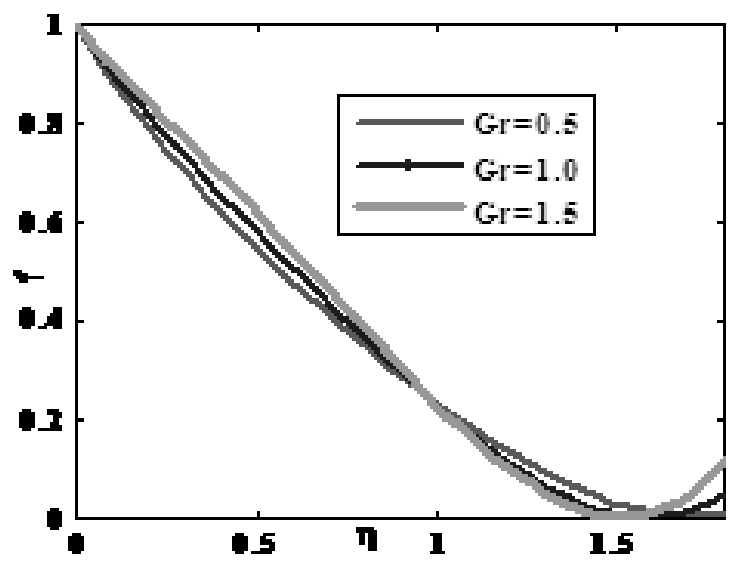

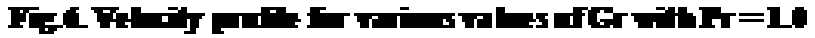
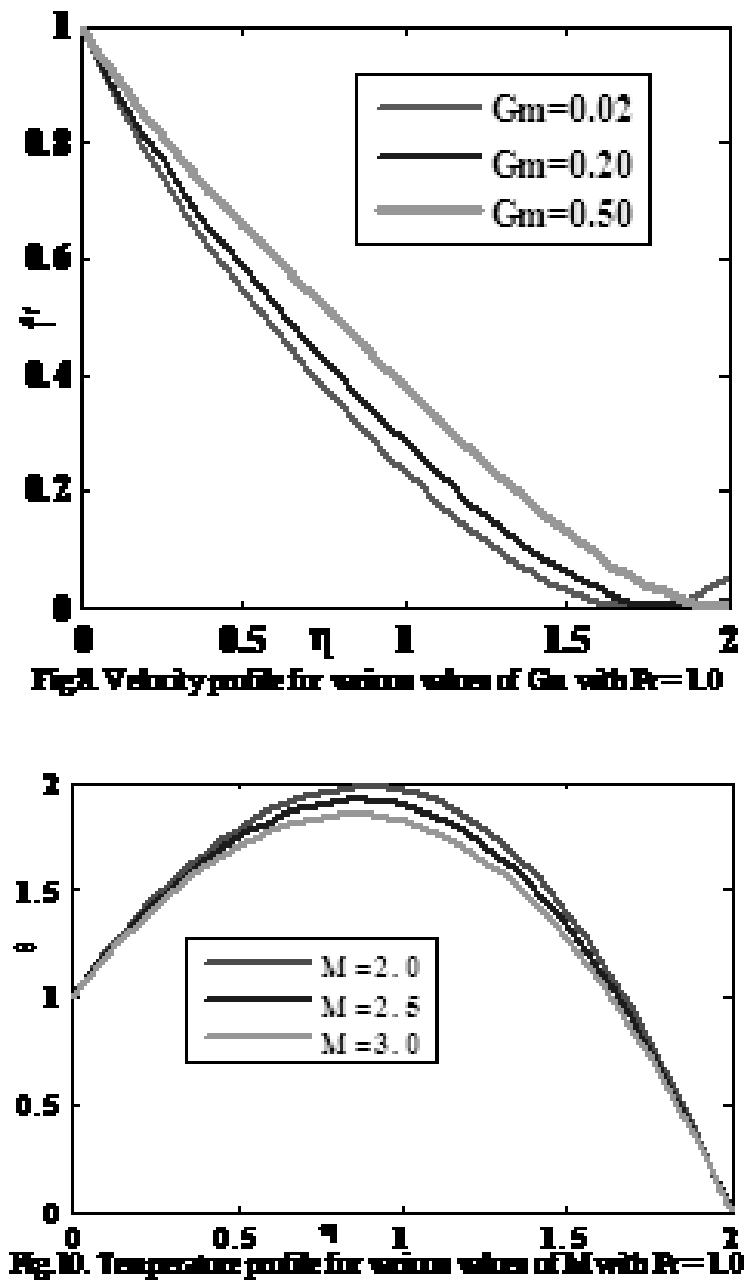
Grashof number both of air and salt water which are shown in Fig.11 - Fig.16. Again, Fig. 17 - Fig. 24 shows the concentration profiles obtained by the numerical simulation for various values of entering non-dimensional parameters. From the Fig. 17 and Fig. 18 it is observed that, the negligible increasing effect on concentration profiles for increasing values of $\mathrm{M}$ in case of air and salt water. Again the significant
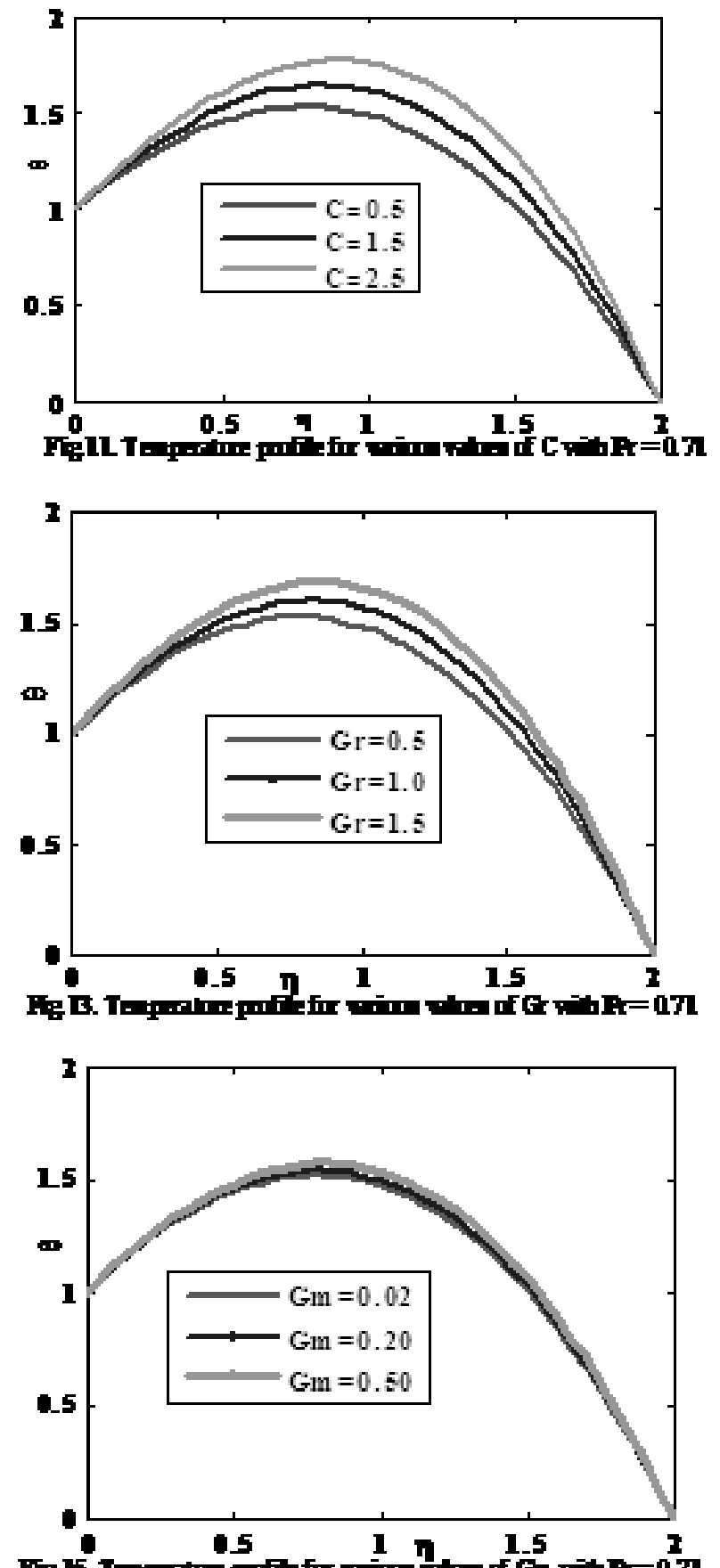

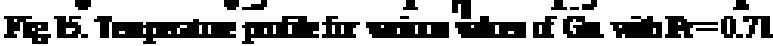

decreasing effect are observed for increasing values of chemical reaction parameter in both of air and salt water which are shown in Fig.19 and Fig.20. Similar result has been found for increasing values of Grashof number but there is no effect for modified Grashof number which are shown in Fig. 21- Fig. 24. Again, from Table I it is observed that the skin friction is decreased for magnetic parameter and

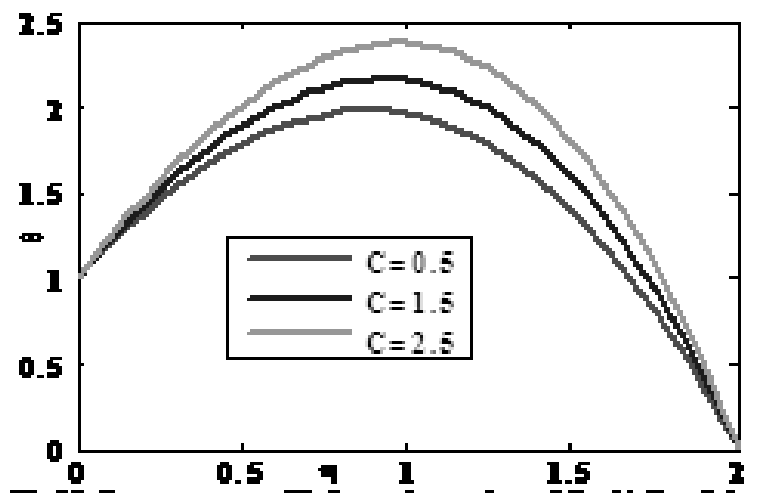

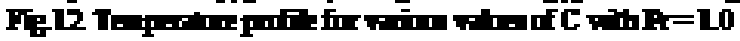
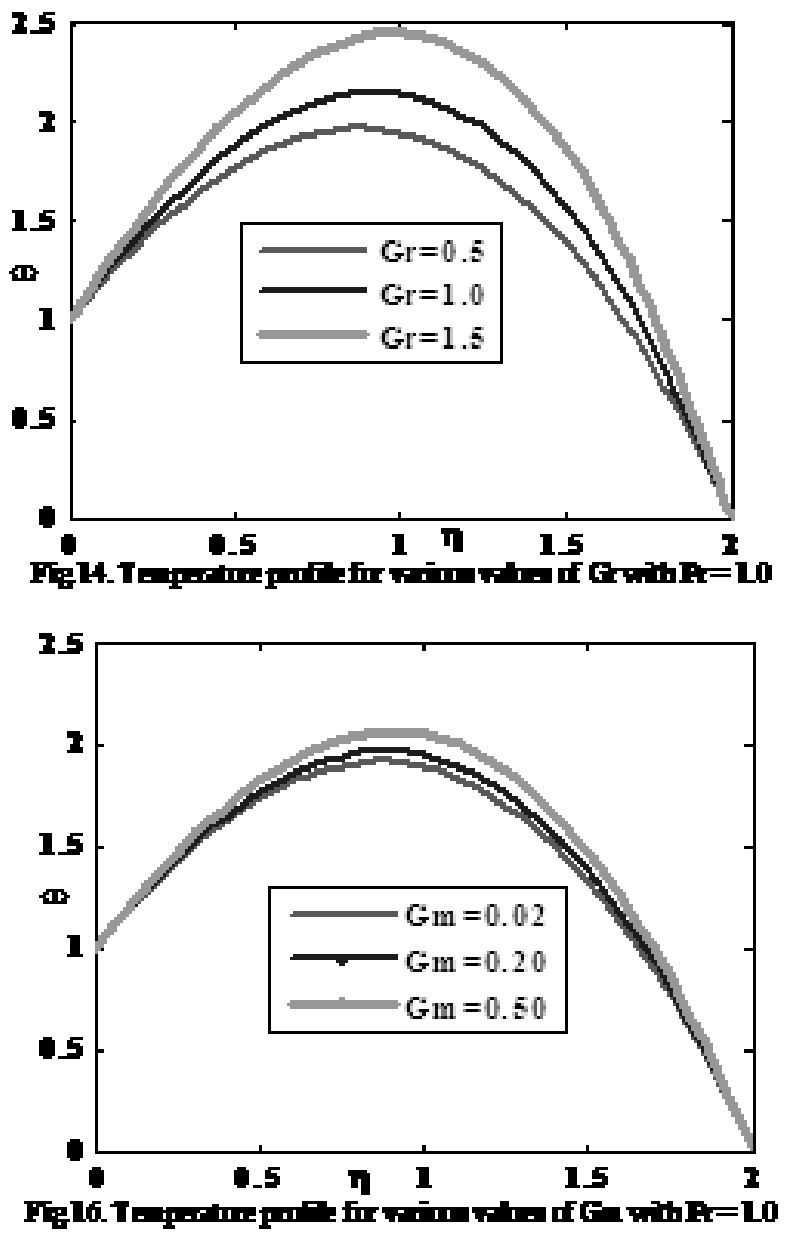

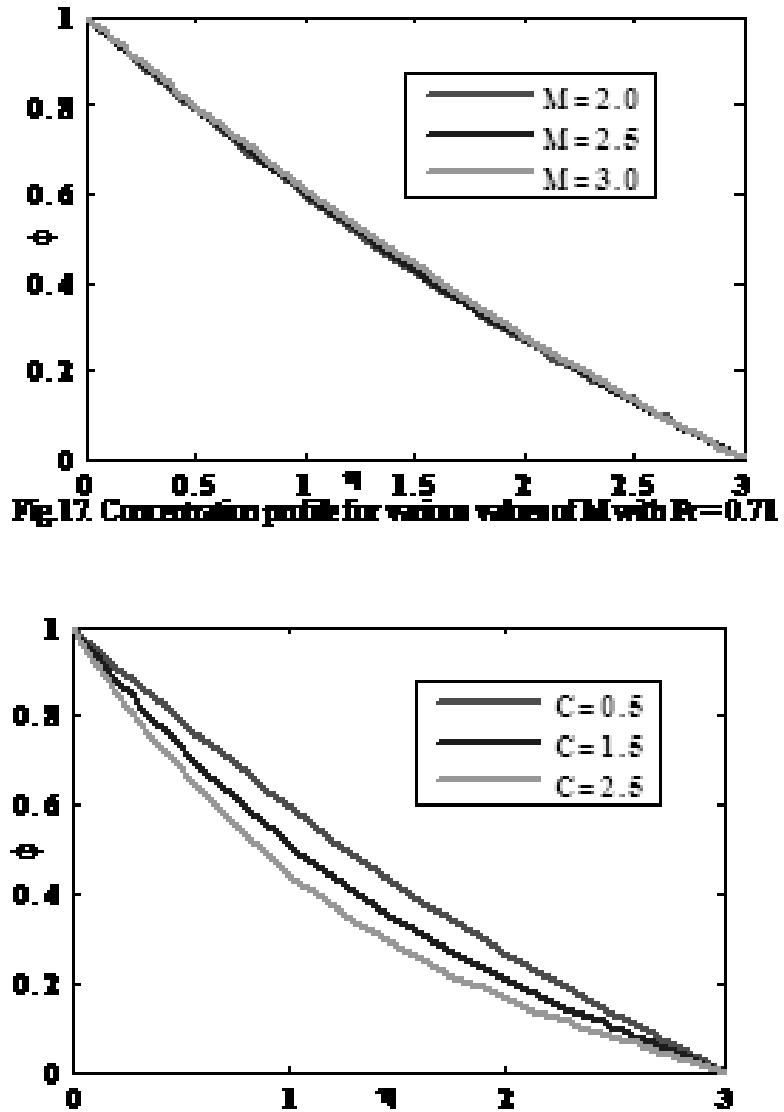

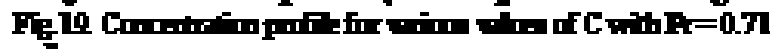

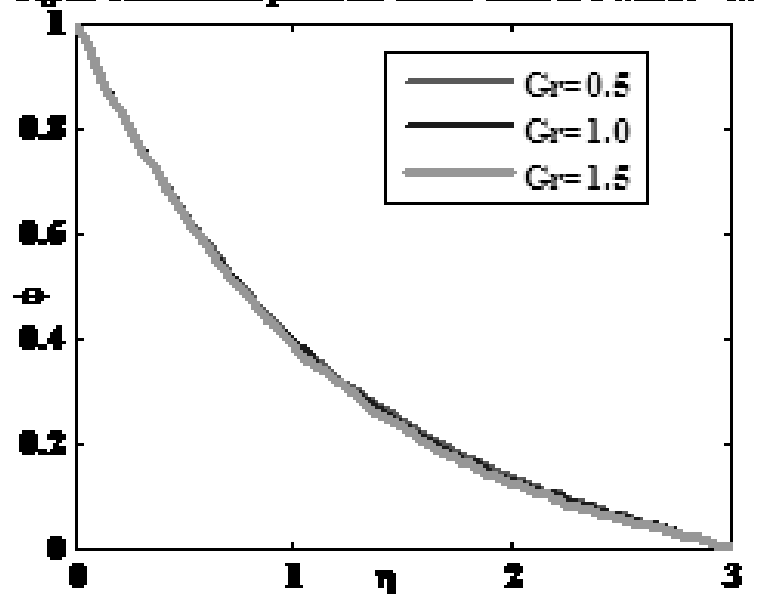

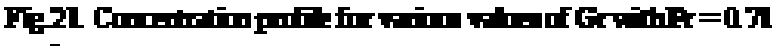

reaction parameter but increased for Gr and Gm in case of air and water as a result the momentum boundary layer is decreased. The rate of heat transfer is increased for magnetic parameter as a result the thermal boundary layer is decreased but reverse trend arises for reaction parameter, Grashof number and modified Grashof number in both of the cases of air and salt water. Again, the rate of concentration is
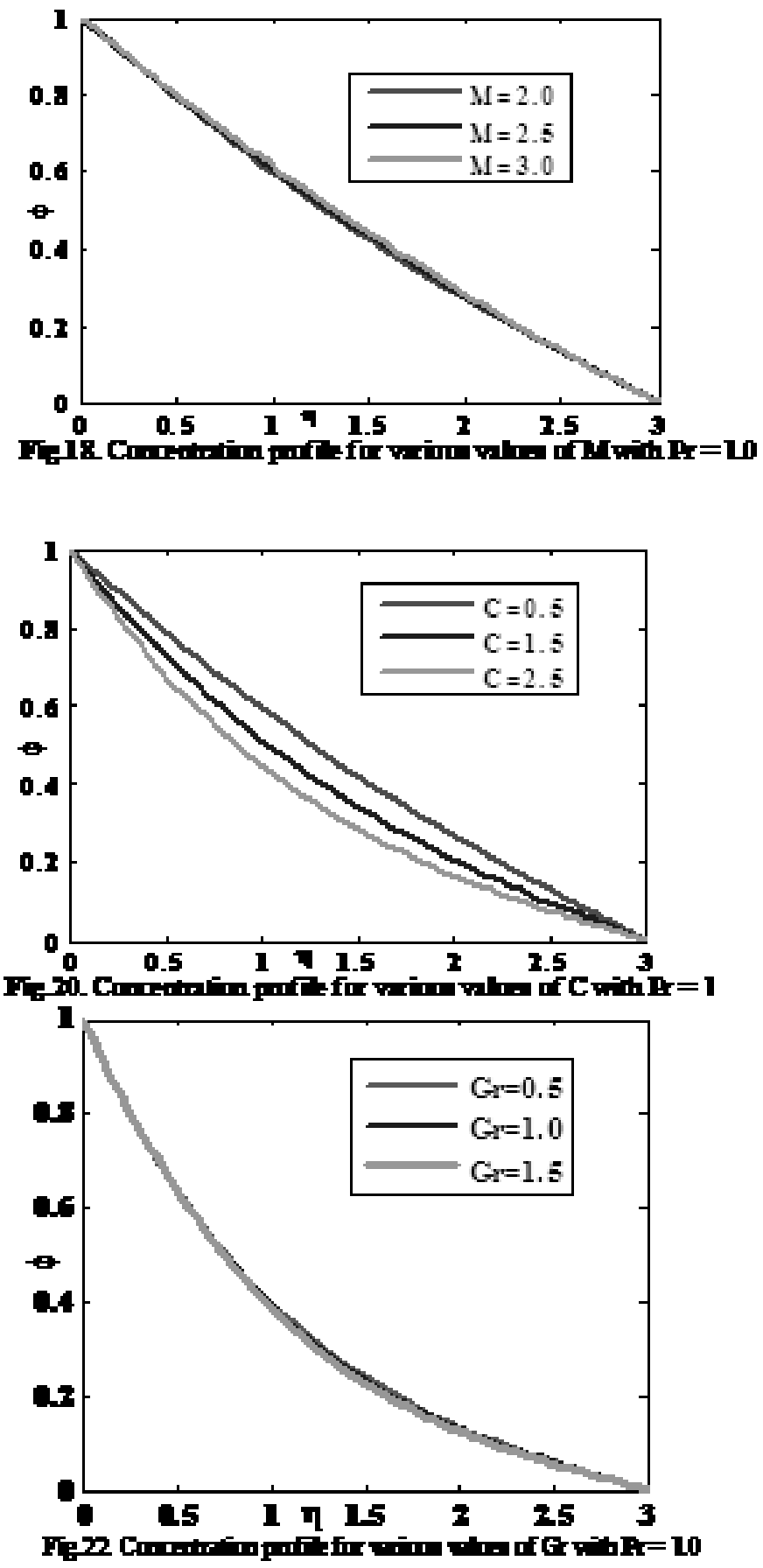

decreased for magnetic parameter but reverse trend arises for chemical reaction parameter, Grashof number and modified Grashof number in both of the cases of air and salt water as a result the concentration boundary layer is increased for $\mathrm{M}$ and decreased for $\mathrm{C}, \mathrm{Gr}$ and $\mathrm{Gm}$. 

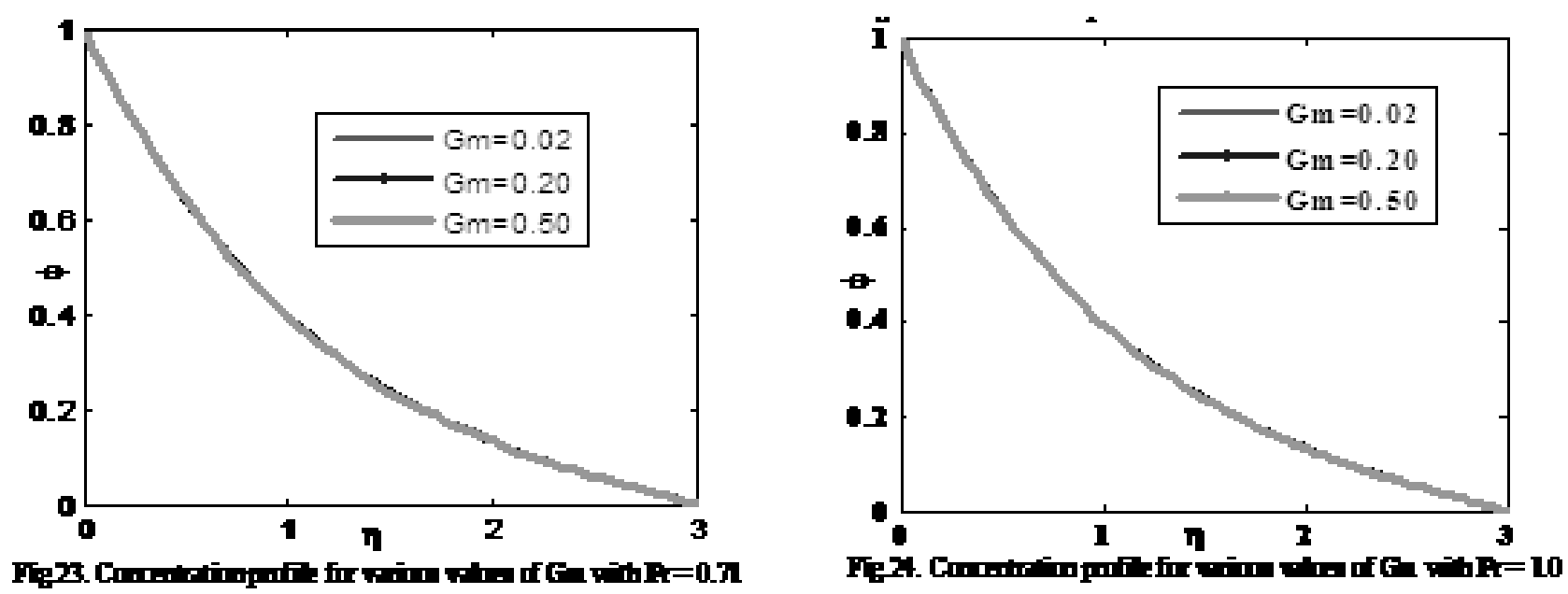

Table I. The shear stress $f^{\prime}(0)$, rate of heat transfer $-\theta^{\prime}(0)$ and rate of concentration $\left(\varphi^{\prime}(0)\right.$ for different values of $\mathrm{M}, \mathrm{C}, \mathrm{Gr}$ and $\mathrm{Gm}$ with $\mathrm{Pr}=0.71 \& 1.0$ are respectively

\begin{tabular}{lccccccccccc}
\hline $\mathrm{M}$ & $\mathrm{C}$ & $\mathrm{Gr}$ & $\mathrm{Gm}$ & \multicolumn{2}{c}{$\mathrm{Pr}$} & \multicolumn{2}{c}{$\mathrm{f}^{\prime}(0)$} & \multicolumn{2}{c}{$\left(\varphi^{\prime}(0)\right.$} & \multicolumn{2}{c}{$-\theta^{\prime}(0)$} \\
\hline 2.0 & 0.5 & 0.5 & 0.02 & 0.71 & 1.0 & -1.1241 & -1.1713 & 0.4231 & 0.4215 & 1.2913 & 2.0778 \\
2.5 & 0.5 & 0.5 & 0.02 & 0.71 & 1.0 & -1.2382 & -1.2803 & 0.4146 & 0.4132 & 1.2479 & 1.9975 \\
3.0 & 0.5 & 0.5 & 0.02 & 0.71 & 1.0 & -1.3447 & -1.3825 & 0.4042 & 0.4023 & 1.2019 & 1.9119 \\
1.0 & 0.5 & 0.5 & 0.02 & 0.71 & 1.0 & -1.0521 & -1.0754 & 0.4412 & 0.4435 & 1.2356 & 1.7965 \\
1.0 & 1.5 & 0.5 & 0.02 & 0.71 & 1.0 & -1.1274 & -1.1754 & 0.5975 & 0.5969 & 1.4363 & 2.2958 \\
0.5 & 2.5 & 0.5 & 0.02 & 0.71 & 1.0 & -1.1309 & -1.1796 & 0.7443 & 0.7439 & 1.5911 & 2.5297 \\
2.0 & 0.5 & 1.0 & 0.02 & 0.71 & 1.0 & -0.9081 & -0.9905 & 0.8772 & 0.8765 & 1.3753 & 2.2791 \\
2.0 & 0.5 & 1.5 & 0.02 & 0.71 & 1.0 & -0.6879 & -0.8017 & 0.8839 & 0.8828 & 1.4799 & 2.6092 \\
2.0 & 0.5 & 0.5 & 0.20 & 0.71 & 1.0 & -0.9943 & -1.0511 & 0.8721 & 0.8718 & 1.3015 & 2.0612 \\
2.0 & 0.5 & 0.5 & 0.50 & 0.71 & 1.0 & -0.8017 & -0.8403 & 0.8741 & 0.8738 & 1.3455 & 2.1779 \\
\hline
\end{tabular}

\section{Conclusions}

The present work has been analyzed in case of air and water for various parameters. From this observation it is seen that the effect of various parameters on velocity, temperature and concentration profiles are almost similar. As, M, increases, the velocity gradient decreases, and leads to enhanced deceleration of the flow for both of air and salt water but reverse trend arises for $\mathrm{Gr}$ and $\mathrm{Gm}$.

* The thermal boundary layer decreases as $M$ increases adjacent to the surface of the plate and the effect is not significant far away from the plate but significant increasing effect are found for reaction parameter, Grashof number and modified Grashof number in case of air and salt water.
* The negligible increasing effect of magnetic parameter and decreasing effect of $\mathrm{Gr} \& \mathrm{Gm}$ on concentration profile but significant decreasing effect for chemical reaction are observed in case of air and water.

\section{References}

Abel MS, Mahesha N (2008), Heat transfer in MHD viscoelastic fluid flow over a stretching sheet with variable thermal conductivity, Non-uniform Heat Source and Radiation, Applied Mathematical Modeling, 32 : 1965-1983.

Afify AA (2004), MHD free-convective flow and mass transfer over a stretching sheet with chemical reaction, Heat and Mass Transfer, 40 : 495-500. 
Alam MS, Ali M, Alim MA, Saha A (2014), Steady MHD boundary free convective heat and mass transfer flow over an inclined porous plate with variable suction and Soret effect in presence of hall current, Bangladesh Journal of Scientific and Industrial Research, 49 : 155-164.

Ali FM, Nazar R, Arifin MN (2010), MHD viscous flow and heat transfer induced by a permeable shrinking sheet with prescribed surface heat flux, WSEAS Transactions on Mathematics, 9: 365-375.

Cortell R (2005), A note on Magnetohydrodynamic flow of a power-law fluid over a stretching sheet, Applied Mathematics and Computation, 168: 557-566.

Ishak A, Nazar R, Pop I (2008), Hydro magnetic flow and heat transfer adjacent to a stretching vertical sheet, Heat Mass Transfer, 44 : 921-927.

Ishak A, Nazar R, Pop I (2006), Unsteady mixed convection boundary layer flow due to a stretching vertical surface, The Arabian Journal for Science and Engineering, 31: 165 - 182.
Rajesh V (2010), MHD effects on free convection and mass transform flow through a porous medium with variable temperature, International Journal of Applied Mathematics and Mechanics, 6: 1 - 16.

Singh PK (2012), Heat and mass transfer in MHD boundary layer flow past an inclined plate with viscous dissipation in porous medium, International Journal of Scientific \& Engineering Research, 3: 1-11.

Sonth SM, Khan SK, Abel MS, Prasad KV (2002), Heat and mass transfer in a visco-elastic fluid flow over an accelerating surface with heat source and viscous dissipation, Heat and Mass Transfer, 38: 213-220.

Tan Y, You XC, Hang Xu, Liao SJ (2008), A new branch of the temperature distribution of boundary layer flows over an impermeable stretching Plate, Heat and Mass Transfer, 44: 501-504.

Received: 15 November 2015; Revised: 17 December 2015; Accepted: 31 January 2016. 